\title{
Transmission of Acceleration from Vibrating Exercise Platforms to the Lumbar Spine and Head
}

\author{
Robert Caryn BA* ${ }^{1,2}$, J.P. Dickey $\mathbf{P h D}^{1.2}$, Alan Salmoni $\mathbf{P h D}^{2}$, Peter Lemon $\mathrm{PhD}^{2}$, Tom J. Hazell $\mathrm{MSc}^{2}$ \\ ${ }^{1}$ Joint Biomechanics Laboratory, ${ }^{2}$ School of Kinesiology, the University of Western Ontario, London, \\ ON
}

\section{Introduction}

Whole Body Vibration platforms have become a popular modality in the fitness and rehabilitation industries. Consequently, whole body vibration has also been identified as a cause of injury in occupational settings ${ }^{2}$. The goal of this study was to quantify the accelerations experienced by the axial skeleton during standing vibration. This study investigated which knee angles effectively dampened vibration to the upper body.

\section{Methods}

Healthy male and female subjects completed whole body vibration trials on a vibrating platform (WAVE) that generated vertical vibrations of 2 or $4 \mathrm{~mm}$ amplitudes between frequency ranges of 20 and $50 \mathrm{~Hz}$. An electrogoniometer (Biometrics SG 150) was used to monitor knee flexion during static squat and dynamic squat trials. Triaxial accelerometers (Biometrics ACL 300/PCB Piezotronics) were placed on the platform surface, lumbar spine (L5) and forehead. A published transfer function was used to calculate bone accelerations from skin accelerations ${ }^{1}$. The magnitude of the accelerations was calculated using root-mean-square (RMS). Transfer functions describing the magnitude and phase frequency response of the skeleton were calculated for the platformto-spine and platform-to-head accelerations.

\section{Results}

Peak vertical accelerations of the platform ranged from 1 to $6.50 \mathrm{~g}$. RMS accelerations experienced at the spine $(0.445 \mathrm{~g})$ and head $(1.01 \mathrm{~g})$ were greatest when the knees were close to full extension, resulting in the greatest transmission of mechanical energy (Fig. 1).

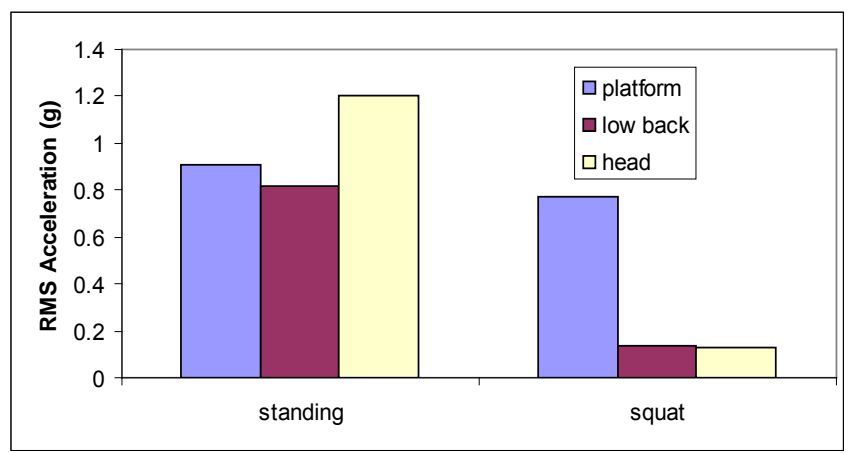

Figure 1. RMS acceleration transmissibility data measured at the head and spine during standing and static squat

\section{Discussion}

The transfer functions illustrated that the body is a nonlinear system (data not presented here). The skeletal acceleration amplitudes showed that the axial skeleton is exposed to large amounts of mechanical energy; full knee extension should be avoided. More research is needed to develop guidelines for safe use of vibrating platforms and to 
explore the long term health effects that may be caused by whole body vibration through the feet.

\section{References}

1. Kitazaki, S., and Griffin, M.J. (1995). A Data Correction Method for Surface Measurement of Vibration on the Human Body. J. of Biomechanics, Vol. 28, No. 7, pp. 885-890.

2. Randall, J.M., Matthews, R.T., and Stiles, M.A. (1997). Resonant Frequencies of Standing Humans. Ergonomics, Vol. 40, No. 9, 879-886. 